\title{
Low-Interference Networks in Metric Spaces of Bounded Doubling Dimension
}

\author{
Anil Maheshwari* Michiel Smid* Norbert Zeh ${ }^{\dagger}$ \\ January 31, 2011
}

\begin{abstract}
Let $S$ be a set of $n$ points in a metric space, let $\mathbf{R}=\left\{R_{p}: p \in S\right\}$ be a set of positive real numbers, and let $G_{\mathbf{R}}$ be the undirected graph with vertex set $S$ in which $\{p, q\}$ is an edge if and only if $|p q| \leq \min \left(R_{p}, R_{q}\right)$. The interference of a point $q$ of $S$ is defined to be the number of points $p \in S \backslash\{q\}$ with $|p q| \leq R_{p}$. For the case when $S$ is a subset of the Euclidean space $\mathbb{R}^{d}$, Halldórsson and Tokuyama have shown how to compute a set $\mathbf{R}$ such that the graph $G_{\mathbf{R}}$ is connected and the maximum interference of any point of $S$ is $2^{O(d)}(1+\log (R / D))$, where $D$ is the closest-pair distance in $S$ and $R$ is the maximum length of any edge in a minimum spanning tree of $S$. In this paper, it is shown that the same result holds in any metric space of bounded doubling dimension. Moreover, it is shown that such a set can be computed in $O(n \log n)$ expected time. It is shown, by an example of a metric space of doubling dimension one, that the upper bound on the maximum interference is optimal. In fact, this example shows that the maximum interference can be as large as $n-1$; in contrast, Halldórsson and Tokuyama have shown that, in $\mathbb{R}^{d}$ where $d \geq 1$ is a constant, there always exists a set $\mathbf{R}$ such that $G_{\mathbf{R}}$ is connected and each point has interference $o\left(n^{2}\right)$.
\end{abstract}

*School of Computer Science, Carleton University, Ottawa, Canada. Research supported by NSERC.

${ }^{\dagger}$ Faculty of Computer Science, Dalhousie University, Halifax, Canada. Research supported by NSERC and the Canada Research Chairs programme. 


\section{Introduction}

Consider a set $S$ of $n$ wireless nodes, which are modeled as points in a metric space. The distance between any two points $p$ and $q$ in $S$ is denoted by $|p q|$. If $p$ is a point in $S$ and $R \geq 0$ is a real number, then $B(p, R)$ denotes the ball with center $p$ and radius $R$. Thus, $B(p, R)=\{q \in S:|p q| \leq R\}$.

Given a set $\mathbf{R}=\left\{R_{p}: p \in S\right\}$ of positive real numbers, define the undirected graph $G_{\mathbf{R}}$ with vertex set $S$ which contains an edge $\{p, q\}$ if and only if $p \neq q, p \in B\left(q, R_{q}\right)$, and $q \in B\left(p, R_{p}\right)$.

The graph $G_{\mathbf{R}}$ represents an ad-hoc wireless network in which $R_{p}$ is the transmission radius of $p$. Two nodes $p$ and $q$ can directly send messages to each other if $p$ is within distance $R_{q}$ of $q$ and $q$ is within distance $R_{p}$ of $p$ (i.e., $\{p, q\}$ is an edge in the network). In order to be useful as a communication network, the set $\mathbf{R}$ must be chosen such that the graph $G_{\mathbf{R}}$ is connected.

Let $q$ be a node in $S$ and consider an edge $\{p, r\}$ in the graph $G_{\mathbf{R}}$ such that $q \in B\left(p, R_{p}\right)$. Since $q$ is within the transmission radius of $p, q$ is affected by communication from $p$ to $r$. Thus, an important issue in wireless networks is limiting the maximum interference. The interference of a node $q$ in $S$ is defined to be the number of nodes $p \neq q$ for which $q$ is within the transmission radius of $p$, i.e.,

$$
\left|\left\{p \in S \backslash\{q\}: q \in B\left(p, R_{p}\right)\right\}\right| .
$$

Let $K(S)$ denote the complete graph with vertex set $S$, and let the weight of each edge $\{p, q\}$ be equal to $|p q|$. Let $D$ be the closest-pair distance in $S$, and let $R$ be the length of a longest edge in a minimum spanning tree of $K(S)$. For the case when $S$ is a subset of $\mathbb{R}^{2}$ and $|p q|$ is the Euclidean distance between $p$ and $q$, Halldórsson and Tokuyama [5] showed how to compute a set $\mathbf{R}$ of transmission radii such that the corresponding graph $G_{\mathbf{R}}$ is connected and the maximum interference of any point of $S$ is $O(1+\log (R / D))$. Their algorithm is based on a quadtree. By using a compressed quadtree and techniques of Arya et al. [2] and Callahan and Kosaraju [4], the set $\mathbf{R}$ can be computed in $O(n \log n)$ time. In fact, this result holds in the Euclidean space $\mathbb{R}^{d}$, for any $d \geq 1$, and the maximum interference of any point is $2^{O(d)}(1+\log (R / D))$.

Halldórsson and Tokuyama conjectured that, again in Euclidean space $\mathbb{R}^{d}$, there always exists a set $\mathbf{R}$ such that $G_{\mathbf{R}}$ is connected and the maximum interference of any point is $O(1+\sqrt{\log (R / D)})$, where the constant hidden in the Big-O may depend on $d$. (This upper bound would be tight, even for 
$d=1$.)

Assouad [3] and Heinonen [7] defined the doubling dimension of a metric space as a generalization of the Euclidean dimension. Let $\lambda$ be the smallest integer such that the following is true: For every real number $\rho>0$, every ball in $S$ of radius $\rho$ can be covered by at most $\lambda$ balls of radius $\rho / 2$. The doubling dimension of the metric space $S$ is defined to be $\log \lambda$, where the base of the logarithm is 2 . It is not difficult to prove that the doubling dimension of the Euclidean metric in $\mathbb{R}^{d}$ is proportional to $d$.

The main purpose of this paper is to give a simple proof for the result of Halldórsson and Tokuyama. As will be shown, this result holds in any metric space of bounded doubling dimension. Thus, the only geometric properties that are needed are the triangle inequality and the fact that every ball can be covered by a "small" number of balls of half the radius. Our main result is the following:

Theorem 1 Let $S$ be a metric space of $n$ points, let $d$ be its doubling dimension, let $D$ be the closest-pair distance in $S$, and let $R$ be the length of a longest edge in a minimum spanning tree of $S$. In $O(n \log n)$ expected time, we can compute a set $\mathbf{R}=\left\{R_{p}: p \in S\right\}$ of positive real numbers such that the graph $G_{\mathbf{R}}$ is connected and each point of $S$ has interference at most $2^{10 d}(2+\lfloor\log (R / D)\rfloor)$.

Observe that in Theorem 1 (as well as in Halldórsson and Tokuyama's result), the upper bound on the maximum interference depends exponentially on the doubling dimension $d$. In Theorem 1 , however, this dependency is very large even for small values of $d$. This is due to the fact that the result is proved by making as few assumptions as possible.

We also show that the conjecture of Halldórsson and Tokuyama is not true for general metric spaces of bounded doubling dimension:

Theorem 2 There exists a metric space $S$ of $n$ points and doubling dimension one such that, for every set $\mathbf{R}=\left\{R_{p}: p \in S\right\}$ of positive real numbers for which the graph $G_{\mathbf{R}}$ is connected, there is a point in $S$ whose interference is $\Omega(1+\log (R / D))$.

Thus, if Halldórsson and Tokuyama's conjecture is true for the Euclidean space $\mathbb{R}^{d}$, its proof must use geometric properties.

Halldórsson and Tokuyama also showed that, in the Euclidean space $\mathbb{R}^{d}$, where $d \geq 1$ is a constant, a set $\mathbf{R}$ can be computed for which the graph 
$G_{\mathbf{R}}$ is connected and every point in $S$ has interference $O(\sqrt{n})$ if $d \leq 2$ and $O(\sqrt{n \log n})$ if $d \geq 3$. The metric space of Theorem 2 shows that this is not true in general metric spaces of bounded doubling dimension: Even if the doubling dimension is equal to one, the maximum interference can be as large as $n-1$.

\section{The proof of Theorem 1}

Throughout this section, $S$ is a metric space of size $n$ and doubling dimension $d$. For the proof of Theorem 1, we need a spanning tree of $S$ that has the following property: For any two points $p$ and $q$ in $S$, every edge on the path in this tree between $p$ and $q$ has length $O(|p q|)$. A minimum spanning tree is an obvious choice for such a tree:

Fact 1 Let $p$ and $q$ be two points in $S$. Every edge on the path between $p$ and $q$ in a minimum spanning tree of $S$ has length at most $|p q|$.

Since the time complexity of computing a minimum spanning tree is $\Theta\left(n^{2}\right)$, even if the doubling dimension is equal to one (see $[6,8]$ ), this would lead to a quadratic-time algorithm. In order to obtain a faster algorithm, we will use a minimum spanning tree of a spanner of $S$.

Recall that, for any real number $t \geq 1$, an undirected graph $H$ with vertex set $S$ is called a $t$-spanner of $S$ if for any two points $p$ and $q$ of $S$, the shortest-path distance between $p$ and $q$ in $H$ is at most $t|p q|$.

Let $H$ be a $t$-spanner of $S$, and let $T$ be a minimum spanning tree of $H$. The following lemma, due to Aronov et al. [1], is a weaker version of Fact 1. Later in this section, we will see that this lemma will be sufficient to prove Theorem 1. In order to be self-contained, a proof of the lemma is included.

Lemma 1 Let $p$ and $q$ be two points in $S$ and consider the path $P$ in $T$ between them. Every edge on $P$ has length at most $t|p q|$.

Proof. Let $\{x, y\}$ be an arbitrary edge on $P$ and assume that $|x y|>t|p q|$. The graph $T^{\prime}$ obtained by removing $\{x, y\}$ from $T$ consists of two components, one containing $p$, the other containing $q$. The $t$-spanner $H$ contains a path $Q$ between $p$ and $q$ having length at most $t|p q|$. The union of $T^{\prime}$ and $Q$ is a connected subgraph of $H$, whose weight is less than the weight of $T$, a contradiction. 
The algorithm for computing the set $\mathbf{R}$ of transmission radii is as follows:

1. Compute a 2-spanner $H$ of $S$ with $O(n)$ edges.

2. Compute a minimum spanning tree $T$ of $H$.

3. For every point $p$ in $S$, compute

$$
R_{p}=\max \{|p q|:\{p, q\} \text { is an edge in } T\} .
$$

4. Return the set $\mathbf{R}=\left\{R_{p}: p \in S\right\}$.

The graph $H$ can be computed in $O(n \log n)$ expected time; see [6]. Since $H$ has $O(n)$ edges, the minimum spanning tree $T$ of $H$ can be computed in $O(n \log n)$ time. Finally, all values $R_{p}, p \in S$, can be computed in $O(n)$ time. Thus, the total expected running time of the algorithm is $O(n \log n)$.

Consider the graph $G_{\mathbf{R}}$ corresponding to the output $\mathbf{R}$ of this algorithm. Observe that each edge $\{p, q\}$ in $T$ is contained in $G_{\mathbf{R}}$. Therefore, this graph is connected.

Let $D$ be the closest-pair distance in $S$, let $R$ be the length of a longest edge in a minimum spanning tree of $K(S)$, and let $L$ be the length of a longest edge in the minimum spanning tree $T$ of the 2 -spanner $H$. The following two lemmas imply that the maximum interference of any point in $S$ is $O(1+\log (R / D))$, completing the proof of Theorem 1 .

Lemma $2 L \leq 2 R$.

Proof. Consider a longest edge $\{p, q\}$ in $T$; thus, $|p q|=L$. Removing this edge from $T$ partitions the set $S$ into two subsets $U$ and $V$. Since $T$ is a minimum spanning tree of $H$, each edge in $H$ connecting a point in $U$ with a point in $V$ has length at least $L$.

We prove that $|u v| \geq L / 2$, for all $u \in U$ and $v \in V$. Since a minimum spanning tree of $S$ has to contain an edge $\{u, v\}$ with $u \in U$ and $v \in V$, this shows that $R \geq L / 2$.

Consider a pair $\{u, v\}$ with $u \in U$ and $v \in V$ and assume for the sake of contradiction that $|u v|<L / 2$. Since $H$ is a 2 -spanner, the shortest path in $H$ between $u$ and $v$ has length less than $L$. This path contains an edge $\{x, y\}$ with $x \in U$ and $y \in V$. Since $\{x, y\}$ belongs to the shortest path between $u$ and $v$, its length is less than $L$. On the other hand, since $x \in U$ and $y \in V$, the length of $\{x, y\}$ is at least $L$, a contradiction. 
Lemma 3 Every point in $S$ has interference at most $2^{10 d}(1+\lfloor\log (L / D)\rfloor)$.

Proof. We may assume, without loss of generality, that $D=1$. Partition the interval $[1, L]$ into subintervals $C_{i}=\left[2^{i}, 2^{i+1}\right), 0 \leq i \leq\lfloor\log L\rfloor$. Let $q$ be a point in $S$. For each $i$ with $0 \leq i \leq\lfloor\log L\rfloor$, define

$$
N_{q}^{i}=\mid\left\{p \in S:|p q| \leq R_{p} \text { and } R_{p} \in C_{i}\right\} \mid .
$$

Then the interference of $q$ is equal to

$$
\left|\left\{p \in S \backslash\{q\}:|p q| \leq R_{p}\right\}\right|=\sum_{i=0}^{\lfloor\log L\rfloor} N_{q}^{i} .
$$

Fix an integer $i$ with $0 \leq i \leq\lfloor\log L\rfloor$. The lemma follows if we can show that $N_{q}^{i} \leq 2^{10 d}$.

Let $k=N_{q}^{i}$ and let $p_{1}, \ldots, p_{k}$ be the points in the set

$$
\left\{p \in S:|p q| \leq R_{p} \text { and } R_{p} \in C_{i}\right\}
$$

For each $j$ with $1 \leq j \leq k$, let $q_{j}$ be a point of $S$ such that $\left\{p_{j}, q_{j}\right\}$ is an edge in $T$ and $\left|p_{j} q_{j}\right|=R_{p_{j}}$.

Let $\rho=2^{i}$ and let $B$ be the ball with center $q$ and radius $4 \rho$. Observe that $\left|q p_{j}\right| \leq R_{p_{j}}<2 \rho$ and

$$
\left|q q_{j}\right| \leq\left|q p_{j}\right|+\left|p_{j} q_{j}\right|=\left|q p_{j}\right|+R_{p_{j}}<4 \rho .
$$

Thus, all points $p_{1}, q_{1}, \ldots, p_{k}, q_{k}$ are contained in $B$.

By applying the definition of doubling dimension five times, we cover $B$ by at most $2^{5 d}$ balls of radius $\rho / 8$. For each $j$ with $1 \leq j \leq k$, since $\left|p_{j} q_{j}\right|=R_{p_{j}} \geq \rho$, the points $p_{j}$ and $q_{j}$ are in different balls of the covering.

Consider any ordered pair $\left(B_{1}, B_{2}\right)$ of balls in the covering of $B$. We prove by contradiction that there is at most one edge in $\left\{\left\{p_{j}, q_{j}\right\}: 1 \leq j \leq k\right\}$ with $p_{j} \in B_{1}$ and $q_{j} \in B_{2}$. This implies that

$$
k \leq 2\left(\begin{array}{c}
2^{5 d} \\
2
\end{array}\right) \leq 2^{10 d}
$$

Assume there are two distinct edges, say, $\left\{p_{1}, q_{1}\right\}$ and $\left\{p_{2}, q_{2}\right\}$, such that $p_{1}, p_{2} \in B_{1}$ and $q_{1}, q_{2} \in B_{2}$; see Figure 1 . Observe that $p_{1} \neq p_{2}$ because 


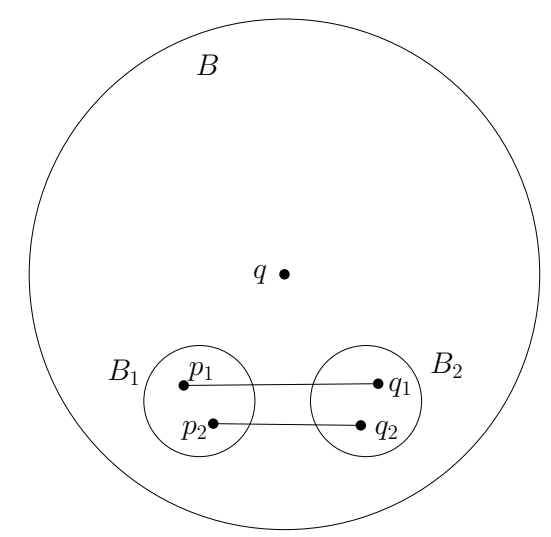

Figure 1: Illustrating the proof of Lemma 3.

each pair $\left\{p_{j}, q_{j}\right\}$ is uniquely determined by $p_{j}$. The points $q_{1}$ and $q_{2}$ are not necessarily distinct.

Let $P$ be the path in $T$ between $p_{1}$ and $p_{2}$. By Lemma 1, each edge on $P$ has length at most $2\left|p_{1} p_{2}\right| \leq \rho / 2<\rho$. Thus, $P$ does not contain $q_{1}$ (otherwise, this path contains the edge $\left\{p_{1}, q_{1}\right\}$, which has length at least $\rho$ ) and $P$ does not contain $q_{2}$ (otherwise, this path contains the edge $\left\{p_{2}, q_{2}\right\}$, which has length at least $\rho$ ). It follows that $q_{1} \neq q_{2}$ (otherwise, $T$ contains a cycle). Let $Q$ be the path in $T$ between $q_{1}$ and $q_{2}$. This path consists of the edge $\left\{p_{1}, q_{1}\right\}$, the path $P$, and the edge $\left\{p_{2}, q_{2}\right\}$. Thus, $Q$ contains the edge $\left\{p_{1}, q_{1}\right\}$, which has length at least $\rho$. This contradicts Lemma 1 , because $\left|q_{1} q_{2}\right|<\rho$.

\section{The lower bound}

In this section, we prove Theorem 2. Let $S=\left\{p_{1}, p_{2}, \ldots, p_{n}\right\}$. For each $i$ and $j$ with $1 \leq i \leq j \leq n$, define

$$
\left|p_{i} p_{j}\right|=\left|p_{j} p_{i}\right|= \begin{cases}0 & \text { if } i=j, \\ 4^{j} & \text { if } i<j .\end{cases}
$$

Smid [8] showed that the doubling dimension of this metric space is equal to one. Observe that for each $j$ with $2 \leq j \leq n$, each of $p_{1}, \ldots, p_{j-1}$ is a nearest neighbor of the point $p_{j}$. 
Consider any set $\mathbf{R}=\left\{R_{p_{j}}: 1 \leq j \leq n\right\}$ of positive real numbers for which the graph $G_{\mathbf{R}}$ is connected. Then, for each $j$ with $2 \leq j \leq n$, $R_{p_{j}} \geq\left|p_{1} p_{j}\right|=4^{j}$ (otherwise, $G_{\mathbf{R}}$ is not connected). It follows that the interference of the point $p_{1}$ is equal to $n-1$.

The closest-pair distance in $S$ is equal to $D=4$, whereas the longest edge in the minimum spanning tree of $S$ has length $R=4^{n}$. Therefore, the interference of $p_{1}$ is

$$
n-1=\log (R / D)=\Omega(1+\log (R / D)) .
$$

This proves Theorem 2 .

\section{Acknowledgement}

Part of this work was done during the Fields Institute Workshop on Discrete and Computational Geometry, Gatineau, Quebec, May 4-8, 2009. The authors thank the participants for fruitful discussions. The authors also thank the three anonymous referees for their helpful comments.

\section{References}

[1] B. Aronov, M. de Berg, O. Cheong, J. Gudmundsson, H. Haverkort, M. Smid, and A. Vigneron. Sparse geometric graphs with small dilation. Computational Geometry: Theory and Applications, 40(3):207-219, 2008.

[2] S. Arya, D. M. Mount, N. S. Netanyahu, R. Silverman, and A. Y. Wu. An optimal algorithm for approximate nearest neighbor searching in fixed dimensions. Journal of the ACM, 45(6):891-923, 1998.

[3] P. Assouad. Plongements lipschitziens dans $\mathbb{R}^{N}$. Bulletin de la Société Mathématique de France, 111:429-448, 1983.

[4] P. B. Callahan and S. R. Kosaraju. A decomposition of multidimensional point sets with applications to $k$-nearest-neighbors and $n$-body potential fields. Journal of the ACM, 42(1):67-90, 1995.

[5] M. M. Halldórsson and T. Tokuyama. Minimizing interference of a wireless ad-hoc network in a plane. Theoretical Computer Science, 402(1):2942, 2008. 
[6] S. Har-Peled and M. Mendel. Fast construction of nets in low-dimensional metrics and their applications. SIAM Journal on Computing, 35(5):11481184, 2006.

[7] J. Heinonen. Lectures on Analysis on Metric Spaces. Springer-Verlag, Berlin, 2001.

[8] M. Smid. The weak gap property in metric spaces of bounded doubling dimension. In Efficient Algorithms, Essays Dedicated to Kurt Mehlhorn on the Occasion of His 60th Birthday, volume 5760 of Lecture Notes in Computer Science, pages 275-289, Berlin, 2009. Springer-Verlag. 